\title{
The cardioprotective effect of dexmedetomidine on elderly patients with cervical cancer in the Trendelenburg position
}

\author{
Jia-Qi Duan ${ }^{1}$, Hou-Gang Huang ${ }^{1, *}$, Chun-Ling Shui ${ }^{1}$, Wei Jiang ${ }^{1}$, Xi Luo ${ }^{1}$
}

${ }^{1}$ Department of Anesthesiology, Yongchuan Hospital of Chongqing Medical University, Yongchuan,

Chongqing province, P. R. China

\section{*Correspondence \\ hhg11112@163.com \\ (Hou-Gang Huang)}

\begin{abstract}
Objectives: To investigate the cardioprotective effect of dexmedetomidine(DEX) on elderly patients with cervical cancer in the Trendelenburg position (TP).

Methods: Eighty patients with cervical cancer presenting with laparoscopic radical hysterectomy (or with laparoscopic pelvic lymphadenectomy) were randomly assigned to the control group (Group C) or the DEX group (Group D), with a total of 40 patients per group. Patients in Group D were given dexmedetomidine, whereas normal saline was administered as placebo in Group C. The data collected for this study included general information (age, body mass index [BMI], anesthesia time, operation time, extubation time and hospital stay), hemodynamic parameters (Heart rate [HR] and mean arterial pressure [MAP]), concentrations of markers of myocardial injury (cardiac troponin I $[\mathrm{cTnI}]$, creatine kinase MB [CK-MB]) and inflammatory factors (Tumor necrosis factor $\alpha[\mathrm{TNF}-\alpha]$, interleukin 6 [IL-6], and C-reactive protein [CRP]) before entering the operating room (T0), $6 \mathrm{~h}$ after the surgery(T9) and $24 \mathrm{~h}$ after the surgery (T10).

Results: Compared to T0, cTnI, CK-MB, TNF- $\alpha$, IL- 6 and CRP were significantly higher at T9 and T10, while cTnI, CK-MB, IL-6 and CRP were significantly lower in group $\mathrm{D}$ than those of group $\mathrm{C}$ at $\mathrm{T} 9$ and $\mathrm{T} 10$. Whilst there was no significant difference in TNF- $\alpha$ between the two groups at T9 $(P=0.821)$, TNF- $\alpha$ concentrations were significantly lower in group D compared to group C at T10 $(P=0.022)$. Extubation time $(P=0.009)$ and hospital stay $(P=0.014)$ were significantly lower in group $\mathrm{D}$, than in group $C$. There was no significant difference in the adverse reactions between the two groups $(P=0.263)$.

Conclusions: In elderly patients with cervical cancer, TP may cause perioperative myocardial damage. DEX could mitigate the damaging effects and show good hemodynamic stability, which is conducive to rapid postoperative resuscitation, and antiinflammatory mechanism may play an important role.
\end{abstract}

\section{Keywords}

Dexmedetomidine; Trendelenburg position; Cervical cancer; Myocardial protection

\section{Introduction}

Cervical cancer is the fourth most common cancer among women worldwide, causing more than 300,000 deaths globally each year. Laparoscopy is preferred over traditional open surgery due to advantages in invasiveness and improved overall prognosis $[1,2]$. In order to optimize the surgical conditions, patients are often placed in a special TP. However, recent studies show that TP can increase cardiac preload and afterload, cardiac oxygen consumption, and the potential of arrhythmias [3], elevating the concentration of postoperative markers of myocardial injury (e.g., CK-MB) [4], which is harmful to the heart. Meanwhile, carbon dioxide $\left(\mathrm{CO}_{2}\right)$, pneumoperitoneum and postural changes can lead to various stress responses such as excessive release of inflammatory factors (IL-6, TNF- $\alpha$, etc.), which in turn can damage the heart directly or indirectly [5]. Especially in elderly patients with poor physical fitness and a high risk of cardiovascular events, the damaging effect can exacerbate the risk of surgery and anesthesia. DEX has been shown to effectively inhibit stress and maintain hemodynamic stability [6]. A number of animal and clinical experiments have confirmed the cardioprotective effect of DEX, however, most of the samples are from patients with cardiac surgery, reports related to TP mainly focus on ocular pressure effects and brain protection, with few clinical studies of cardioprotective effects in cervical cancer patients. Therefore, in this study, markers of myocardial injury and inflammatory factors were measured at predefined time points to investigate the cardioprotective effect of DEX. 


\section{Subjects and methods}

This double-blind randomised control trial was approved by the Yongchuan Hospital of Chongqing Medical University, and was registered at http://www.chictr.org.cn (Chinese Clinical Trial Registry, ChiCTR2000030319). The study was conducted from October 2019 to June 2020 at Yongchuan Hospital of Chongqing Medical University. After obtaining written informed consents from patients, 80 patients with cervical cancer who undergoing laparoscopic radical hysterectomy (or laparoscopic pelvic lymphadenectomy) were selected. The inclusion criteria was as follows: age greater than or equal to 65 years; $18.5 \mathrm{~kg} / \mathrm{m}^{2}<$ BMI $<29.9 \mathrm{~kg} / \mathrm{m}^{2}$; and classification by the American Society of Anesthesiologists as Class I-III. Patients were excluded if their medical history included any of the following: myocardial infarction (MI), and/or coronary artery disease (CAD) with any abnormality in the electrocardiogram; allergic reaction to DEX. Sample size was estimated by PASS 15.0 software (NCSS Inc., USA) and based on the Xu's and Zhou's studies $[7,8]$. Power calculations, with significance level of $\alpha=0.05$ and test efficacy $(1-\beta)=0.9$, showed that it was necessary to recruit 31 patients per group. Considering best practices and utilization of experimental materials and an expected dropout rate of $20 \%$, the study aimed to recruit 40 patients per group.

According to computer-generated randomization, patients were randomly assigned to one of two groups: DEX group (Group D, N = 40) and control group (Group C, N = 40). Patients of Group D received a loading dose of $0.5 \mu \mathrm{g} \cdot \mathrm{kg}^{-1}$ DEX $10 \mathrm{~min}$ before the operation, followed by an infusion at the rate of $0.5 \mu \mathrm{g} \cdot \mathrm{kg}^{-1} \cdot \mathrm{h}^{-1}$ until $30 \mathrm{~min}$ before the end of the surgery. Patients in Group C in contrast, received a normal saline as placebo. Patients were fasted for $8 \mathrm{~h}$ before surgery and basic vital signs (HR, MAP, electrocardiogram, pulse oxygen saturation [SpO2], etc.) were collected with Patient Monitor (Mindray Benevision N12), and bispectral index was obtained using Depth of Anesthesia Monitor (Taiji Medical Technology Co.TD-3200A). All patients were intubated after induction of anesthesia (Tracheal tube I.D. 7.0), intraoperative fluid therapy was combined with crystalloid and colloid fluids. Patients were pre-infused with an infusion of sodium lactate Ringer's injection $10 \mathrm{~mL} / \mathrm{kg}$ as a loading dose prior to induction of anesthesia, followed by a background dose of $5 \mathrm{~mL} \cdot \mathrm{kg}^{-1} \cdot \mathrm{h}^{-1}$, which was adjusted to maintain MAP $>80 \mathrm{mmHg}$ and urine output $>0.5 \mathrm{~mL} \cdot \mathrm{kg}^{-1} \cdot \mathrm{h}^{-1}$. When intraoperative blood loss $<$ $500 \mathrm{~mL}$, fluid volume was supplemented with an equal dose of $6 \%$ hydroxyethyl starch 130/0.4. Anesthesia was maintained with remifentanil, propofol, and atracurium, with the BIS value kept between 40 and 60 . The pneumoperitoneum pressure was maintained at $12 \mathrm{mmHg}$, and all laparoscopic operations and anesthesia were performed by the same group of surgeons and anesthesiologists.

HR and MAP were measured before entering the operating theatre (T0), at the beginning of the anesthesia (T1), at the beginning of the surgery (T2), when shifting from supine to TP (T3), 30 min after placing patients in the TP (T4), $1 \mathrm{~h}$ after placing patients in the TP (T5), at return to the supine position (T6), at the end of the surgery (T7), and after extubation (T8). Venous blood samples $(4 \mathrm{~mL})$ were taken from all patients at
T0, $6 \mathrm{~h}$ after the surgery (T9) and $24 \mathrm{~h}$ after the surgery (T10). Extubation time (time passed from the end of the surgery until extubation) and the length of hospital stay were also recorded. Extubation standards were consistent with DAS Extubation Guidelines (UK, 2012). Samples were centrifuged at $4{ }^{\circ} \mathrm{C}$ (3000 $\mathrm{r} \cdot \mathrm{min}^{-1}, 10 \mathrm{~min}$ ) and the supernatant was subsequently stored at $-80{ }^{\circ} \mathrm{C}$. Concentrations of serum cTnI, CK-MB, TNF- $\alpha$, IL-6, and CRP were determined using enzyme-linked immunosorbent assay (ELISA).

\subsection{Statistical analysis}

Data were processed using SPSS 19.0 software (SPSS Inc., Chicago, IL, USA), data of general information (age, BMI, anesthesia time, operation time, extubation time and hospital stay) and the concentrations of markers of myocardial injury (cTnI, CK-MB) and inflammatory factors (TNF- $\alpha$, IL-6, and CRP) were expressed as mean \pm standard deviation, using $t$ test; adverse reactions were expressed as a percentage, using Fisher's Exact test, and $P$-value $<0.05$ indicated statistical significance.

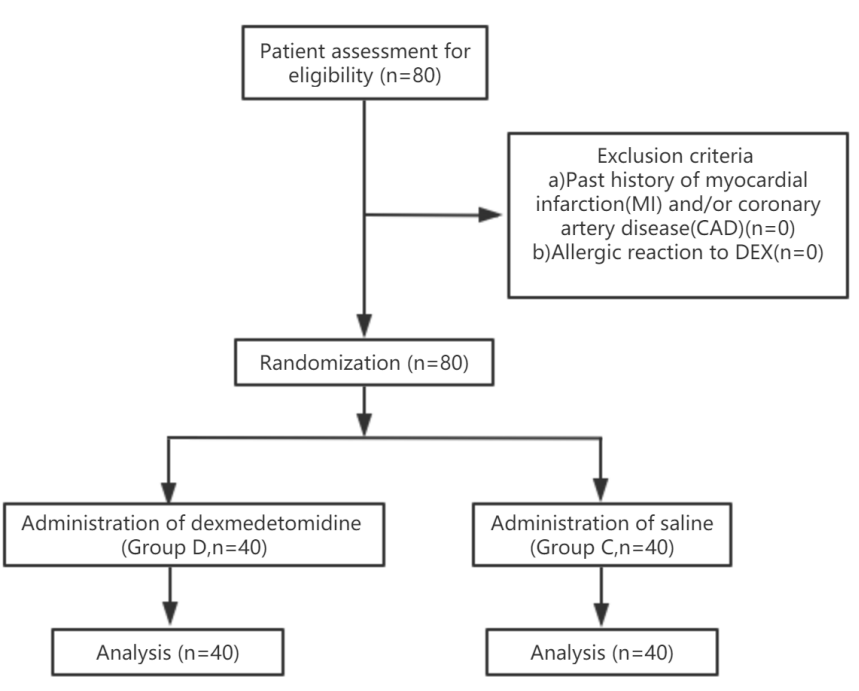

F I G U RE 1. Flow diagram of the study.

TA B L E 1. Patient demographics.

$\begin{array}{lccc}\text { General information } & \text { Group C } & \text { Group D } & P \text {-value } \\ \text { Age }(\text { years }) & 69.78 \pm 4.45 & 70.25 \pm 4.13 & 0.622 \\ \text { BMI }\left(\mathrm{kg} / \mathrm{m}^{2}\right) & 24.00 \pm 2.65 & 25.08 \pm 2.48 & 0.063 \\ \text { Anesthesia time (min) } & 259.02 \pm 32.44 & 266.13 \pm 38.37 & 0.374 \\ \text { Operation time (min) } & 236.25 \pm 32.75 & 239.13 \pm 38.76 & 0.721 \\ \text { Extubation time (min) } & 39.03 \pm 11.35 & 32.08 \pm 11.90 & 0.009 \\ \text { Hospital stay (d) } & 6.23 \pm 2.32 & 5.10 \pm 1.61 & 0.014\end{array}$

\section{Results}

\subsection{General information}

Eighty patients (40 per group) were recruited from October 2019 to June 2020 (Fig. 1). There were no statistically significant group differences with respect to age, BMI, time 
of anesthesia or surgery (Table 1$)$. Extubation time $(P=$ $0.009)$ and length of hospital stay $(P=0.014)$ were however significantly shorter in group D compared to group C (Table $1)$.

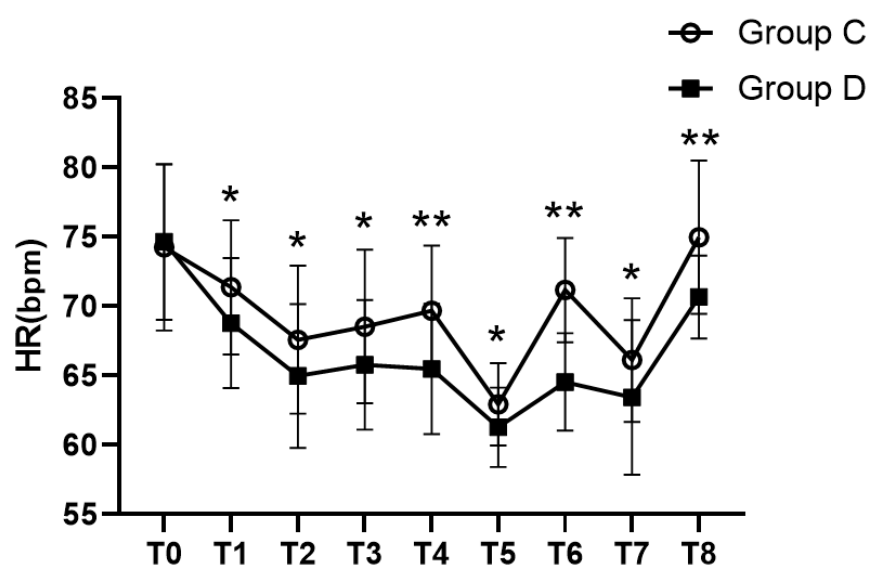

F I G URE 2. Comparison of HR between the groups at T0-T8. $* P<0.05 ; * * P<0.001$.

\subsection{Hemodynamic parameters}

There was no significant difference in HR and MAP between the two groups at T0. At T1-T8, HR and MAP were significantly lower in group D compared to group C. Fluctuations in HR and MAP were less pronouned in group D across all time points (Fig. 2 and Fig. 3).

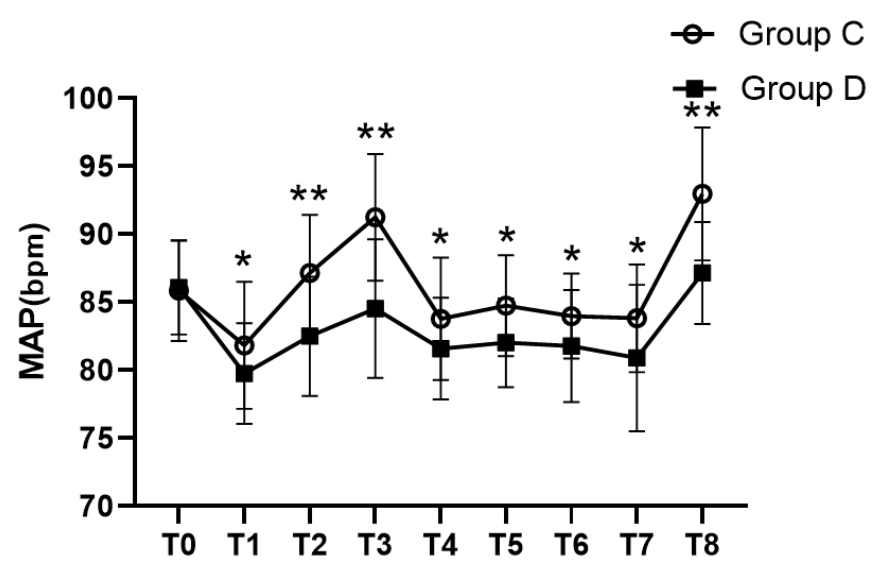

F I G U R E 3. Comparison of MAPs between the groups at T0-T8. ${ }^{*} P<0.05 ; * * P<0.001$.

\subsection{Markers of myocardial injury}

There was no significant difference in CK-MB or cTnI between the two groups at T0 (Table 2). The level of CK-MB and cTnI increased significantly in both groups when comparing T9 and T10 against T0 $(P<0.001)$, and they were significantly lower in group D than that in group C at T9 and T10 (Table 2).

\subsection{Inflammatory factors}

There was no significant difference in TNF- $\alpha$, IL-6 or CRP between the two groups at T0 (Table 3 ). Compared with T0,
TA B L E 2. Comparison of serum levels of CK-MB and cTnI between the groups at different time points.

Markers of myocardial
injury Group C Group D $P$-value

CK-MB (ng/mL)

\begin{tabular}{lccc} 
T0 & $3.72 \pm 2.09$ & $3.55 \pm 2.27$ & 0.729 \\
T9 & $32.14 \pm 4.54$ & $20.66 \pm 3.27<0.001$ \\
T10 & $40.60 \pm 3.87$ & $24.10 \pm 3.72<0.001$ \\
cTnI $(\mathrm{ng} / \mathrm{mL})$ & & & \\
T0 & $0.25 \pm 0.20$ & $0.25 \pm 0.13$ & $>0.999$ \\
T9 & $2.31 \pm 0.74$ & $1.86 \pm 0.67$ & 0.006 \\
T10 & $2.50 \pm 0.68$ & $2.06 \pm 0.66$ & 0.004 \\
\hline
\end{tabular}

the level of TNF- $\alpha$, IL- 6 and CRP increased significantly in both groups at T9 and T10 $(P<0.001)$, and IL- 6 and CRP were significantly lower in group D compared to group $\mathrm{C}$ at $\mathrm{T} 9$ and T10 (Table 3). There was no significant difference in TNF$\alpha$ between the two groups at T9, whereas at T10 the level of TNF- $\alpha$ were significantly lower in group D compared to group C (Table 3).

TA B L E 3. Comparison of serum levels of inflammatory factors between the two groups at T0, T9, and T10.

$\begin{array}{lccc}\text { inflammatory factors } & \text { Group C } & \text { Group D } & P \text {-value } \\ \text { IL-6 }(\mathrm{pg} / \mathrm{mL}) & & & \\ \text { T0 } & 43.33 \pm 7.75 & 44.02 \pm 8.78 & 0.712 \\ \text { T9 } & 62.94 \pm 9.51 & 51.36 \pm 8.44<0.001 \\ \text { T10 } & 72.49 \pm 9.77 & 61.67 \pm 7.99<0.001 \\ \text { TNF- } \alpha(\mathrm{pg} / \mathrm{mL}) & & & \\ \text { T0 } & 20.50 \pm 7.51 & 21.26 \pm 6.50 & 0.632 \\ \text { T9 } & 36.19 \pm 8.53 & 36.61 \pm 8.16 & 0.821 \\ \text { T10 } & 50.48 \pm 7.45 & 46.44 \pm 8.03 & 0.022 \\ \text { CRP }(\mathrm{mg} / \mathrm{L}) & & & \\ \text { T0 } & 2.36 \pm 3.05 & 1.92 \pm 2.87 & 0.508 \\ \text { T9 } & 9.22 \pm 8.87 & 5.46 \pm 4.69 & 0.020 \\ \text { T10 } & 13.80 \pm 15.72 & 6.97 \pm 8.44 & 0.018\end{array}$

\subsection{Adverse reactions}

There was no significant difference in adverse reactions between the two groups (Table 4).

\section{Discussion}

In this randomized double-blinded controlled trial, administration of DEX led to a reduction in the concentration of CK-MB and cTnI, 6 and 24 hours postoperatively, as well as the release of inflammatory factors (IL-6, CRP, etc.).

Currently, accepted cardioprotective mechanisms of DEX include anti-sympathetic, inhibition of apoptosis, inhibition of oxidative stress, inflammatory responses, and other pathways. In the present study, it was found that the HR and MAP 
TA B L E 4. Comparison of adverse reactions of the patients in the two groups.

$\begin{array}{lccc}\text { Adverse reactions } & \text { Group C } & \text { Group D } & P \text {-value } \\ \text { Nausea and vomiting } & 3(7.5 \%) & 1(2.5 \%) & / \\ \text { Postoperative irritability } & 1(2.5 \%) & 1(2.5 \%) & / \\ \text { Chocking } & 2(5.0 \%) & 0 & / \\ \text { Total } & 6(15.0 \%) & 2(5.0 \%) & 0.263\end{array}$

were significantly lower in group D compared to group $\mathrm{C}$ at each time point after anesthesia $(P<0.05)$, and fluctuations in HR and MAP were less pronouned in group D across all time points. DEX could therefore effectively reduce the stress brought about by stimuli such as postural changes, intubation and skin incision. In both groups, cTnI and CK-MB were significantly increased at $6 \mathrm{~h}$ and $24 \mathrm{~h}$ postoperatively $(P<$ 0.001). In group $\mathrm{D}, \mathrm{cTnI}$ and CK-MB were significantly reduced at T9 and T10 after surgery compared with group $\mathrm{C}$ $(P<0.05)$, indicating that DEX might confer cardioprotective effects. In the study, the adoption of TP increases cardiac preload due to gravity, increased cardiac blood flow, and increased cardiac afterload due to increased MAP and systemic vascular resistance(SVR) [3, 4], both of which increase cardiac oxygen consumption. DEX reduces sympathetic nervous system tension by highly selective binding of $\alpha 2$ adrenergic receptors in the central nervous system and peripheral vasculature [9], resulting in a significant reduction in HR and MAP, thereby reducing cardiac work to reduce cardiac oxygen consumption. In addition, DEX reduces serum catecholamine concentrations by inhibiting multiple stress responses (postural changes, intubation, etc.) [9], maintains hemodynamic stability, and improves the balance between oxygen supply and demand. Nadir et al. (2015) also found a significant increase in lactate dehydrogenase (LDH), CK, and CK-MB at $6 \mathrm{~h}$ postoperatively in patients treated with TP [10], indicating that TP can cause some degree of myocardial damage, but they did not further investigate how to mitigate the damaging effects. The present study fills in the gap. Tang et al. (2018) found that DEX could reduce perioperative stress and adverse reactions, helping expedite rapid patients recovery [11], Miao et al. (2020) concluded that DEX could reduce the time to extubation and also the hospital stay [12], both contributing to rapid postoperative recovery.

In terms of suppressing the inflammatory response, this study found that the postoperative inflammatory factors IL6 , TNF- $\alpha$ and CRP were significantly elevated in both groups $(P<0.05)$, which may be related to stressors such as surgical treatment, and the immune and inflammatory responses of the body. Chen et al. (2017) also found a significant increase in the release of inflammatory factors at the end of surgery in liver transplant patients, with elevated markers of myocardial injury lasting up to $24 \mathrm{~h}$ postoperatively [13]. Of the above inflammatory factors, IL-6 may act as a predictor of dangerous cardiovascular events, exerting negative inotropic and cytotoxic effects, causing structural and functional impairment of the left ventricle $[14,15]$. TNF- $\alpha$ usually appears in the early stages of the inflammatory response and can stimulate the production of inflammatory factors such as IL-6, triggering a sustained inflammatory response [16], thus their release can directly or indirectly damage the heart. In the present study, the inflammatory factors were significantly lower in group D than in group $\mathrm{C}$ at $6 \mathrm{~h}$ and $24 \mathrm{~h}$ postoperatively $(P<0.05)$. This may be due to the fact that DEX reduces the release of inflammatory factors by activating alpha-2 adrenergic receptors, activating cholinergic transmission and reducing the stress response [17]. In addition, DEX also affects immune cells, shifting their cytokine gene expression from pro-inflammatory to antiinflammatory [18]. Animal experiments revealed that DEX can reduce inflammation and protect myocardium by inhibiting high mobility family protein B1 (HMGB1), downregulating Toll-like receptor 4 (TLR4)-myeloid differentiation factor 88 (MyD88)-NF- $\kappa$ B signaling pathway, reducing the release of inflammatory factors such as IL-6 and TNF- $\alpha$, and decreasing serum cTnI and CK-MB concentrations [19, 20]. Therefore, it confirmed that the anti-inflammatory effect of DEX is important for cardioprotection, which is consistent with the present study, but the specific mechanism is still unclear and requires further study.

\section{Conclusions}

In elderly patients with cervical cancer, TP may cause perioperative myocardial damage. DEX could mitigate the damaging effects and show good hemodynamic stability, which is conducive to rapid postoperative resuscitation, and antiinflammatory mechanism may play an important role.

\section{AUTHOR CONTRIBUTIONS}

Jiaqi Duan and Hougang Huang designed the study. Chunling Shui, Xi Luo and Wei Jiang collected and analyzed the data. Jiaqi Duan analyzed the results and drafted the manuscript. Hougang Huang revised the manuscript and coordinated the entire experiment.

\section{ETHICS APPROVAL AND CONSENT TO PARTICIPATE}

This double-blind randomised control trial was approved by the Yongchuan Hospital of Chongqing Medical University, and was registered at http://www.chictr.org.cn (Chinese Clinical Trial Registry, ChiCTR2000030319).

\section{ACKNOWLEDGMENT}

The authors acknowledge the institutional support from Yongchuan Hospital of Chongqing Medical University.

\section{FUNDING}

Project of the Science and Technology Committee of Yongchuan District, Chongqing province, China (Ycstc, 2015nc50002), Postgraduate Innovation Fund of Yongchuan Hospital of Chongqing Medical University (YJSCX201911). 


\section{CONFLICT OF INTEREST}

The authors declare no conflict of interest.

\section{REFERENCES}

[1] Ward ZJ, Grover S, Scott AM, Woo S, Salama DH, Jones EC, et al. The role and contribution of treatment and imaging modalities in global cervical cancer management: survival estimates from a simulation-based analysis. The Lancet Oncology. 2020; 21: 1089-1098.

[2] Bhave Chittawar P, Franik S, Pouwer AW, Farquhar C. Minimally invasive surgical techniques versus open myomectomy for uterine fibroids. Cochrane Database of Systematic Reviews. 2014; CD004638.

[3] Atkinson TM, Giraud GD, Togioka BM, Jones DB, Cigarroa JE. Cardiovascular and ventilatory consequences of laparoscopic surgery. Circulation. 2017; 135: 700-710.

[4] Arvizo C, Mehta ST, Yunker A. Adverse events related to Trendelenburg position during laparoscopic surgery. Current Opinion in Obstetrics and Gynecology. 2018; 30: 272-278.

[5] Naqvi SEH, Zaka-Ur-Rab A, Islam N, Ali E. A prospective study of altered inflammatory response and its clinical outcome following laparoscopic and open cholecystectomy. Iranian Journal of Medical Sciences. 2017; 42: 347-353.

[6] Wu XM, Xue ZG, Ma H. Expert consensus on clinical application of dexmedetomidine. Journal of Anesthesiology. 2018; 34: 820-823.

[7] $\mathrm{Xu} \mathrm{L}, \mathrm{Hu} \mathrm{Z}$, Shen J, McQuillan PM. Does dexmedetomidine have a cardiac protective effect during non-cardiac surgery? A randomised controlled trial. Clinical and Experimental Pharmacology and Physiology. 2014; 41: 879-883.

[8] Zhou H, Zhou D, Lu J, Wu C, Zhu Z. Effects of pre-cardiopulmonary bypass administration of dexmedetomidine on cardiac injuries and the inflammatory response in valve replacement surgery with a sevoflurane postconditioning protocol. Journal of Cardiovascular Pharmacology. 2019; 74: 91-97.

[9] Funai Y, Pickering AE, Uta D, Nishikawa K, Mori T, Asada A, et al. Systemic dexmedetomidine augments inhibitory synaptic transmission in the superficial dorsal horn through activation of descending noradrenergic control: an in vivo patch-clamp analysis of analgesic mechanisms. Pain. 2014; 155: 617-628.

[10] Sinikoglu NS, Gumus F, Sanli N, Totoz T, Alagol A, Turan N. Cardiac and liver marker alterations after laparoscopic gynaecologic operations. Turkish Journal of Anesthesia and Reanimation. 2015; 43: 73-77.

[11] Xia Z, Tang C, Li J, Zhang Z, Zhao B, Wang S, et al. Neuroprotective effect of bispectral index-guided fast-track anesthesia using sevoflurane combined with dexmedetomidine for intracranial aneurysm embolization. Neural Regeneration Research. 2018; 13: 280-288.

[12] Miao M, Xu Y, Li B, Chang E, Zhang L, Zhang J. Intravenous administration of dexmedetomidine and quality of recovery after elective surgery in adult patients: a meta-analysis of randomized controlled trials. Journal of Clinical Anesthesia. 2020; 65: 109849.

[13] Chen JB, Chen GY, Wei SD, Sun JJ, Liu GB, Xie ZT, et al. Inflammatory factor in donor liver and its effect on recipient myocardial injury after liver transplantation. European Review for Medical and Pharmacological Sciences. 2017; 21: 4362- 4368.

[14] Wu YJ, Wang J. Relationship between IL-6 level and myocardial remodelling in patients with CHF. Chinese Journal of General Practice. 2014; 12: 378-379.

[15] Rodondi N, Marques-Vidal P, Butler J, Sutton-Tyrrell K, Cornuz J, Satterfield S, et al. Markers of atherosclerosis and inflammation for prediction of coronary heart disease in older adults. American Journal of Epidemiology. 2010; 171: 540-549.

[16] Miao Z, Wu P, Wang J, Zhou FC, Lin Y, Lu XY, et al. Wholecourse application of dexmedetomidine combined with ketorolac in nonnarcotic postoperative analgesia for patients with lung cancer undergoing thoracoscopic surgery: a randomized control trial. Pain Physician. 2020; 23: E185-E193.

[17] Xiang H, Hu B, Li Z, Li J. Dexmedetomidine controls systemic cytokine levels through the cholinergic anti-inflammatory pathway. Inflammation. 2014; 37: 1763-1770.

[18] Romero-Sandoval EA, McCall C, Eisenach JC. Alpha2-adrenoreceptor stimulation transforms immune responses in neuritis and blocks neuritisinduced pain. The Journal of Neuroscience. 2005; 25: 8988-8994.

[19] Zhang JJ, Peng K, Zhang J, Meng XW, Ji FH. Dexmedetomidine preconditioning may attenuate myocardial ischemia/reperfusion injury by down-regulating the HMGB1-TLR4-MyD88-NF-кB signaling pathway. PLoS ONE. 2017; 12: e0172006.

[20] Chen J, Jiang Z, Zhou X, Sun X, Cao J, Liu Y, et al. Dexmedetomidine preconditioning protects cardiomyocytes against hypoxia/reoxygenationinduced necroptosis by inhibiting HMGB1-mediated inflammation. Cardiovascular Drugs and Therapy. 2019; 33: 45-54.

How to cite this article: Jia-Qi Duan, Hou-Gang Huang, Chun-Ling Shui, Wei Jiang, Xi Luo. The cardioprotective effect of dexmedetomidine on elderly patients with cervical cancer in the Trendelenburg position. Signa Vitae. 2021;17(5):117-121. doi:10.22514/sv.2021.007. 